\title{
Carnets
}

Revue électronique d'études françaises de l'APEF

Deuxième série - 19 | 2020

Petite fabrique d'interprètes

\section{La question du roman terraqué aujourd'hui}

\section{Nathalie Roelens}

\section{(2) OpenEdition}

Journals

Édition électronique

URL : http://journals.openedition.org/carnets/11782

DOI : 10.4000/carnets. 11782

ISSN : 1646-7698

Éditeur

APEF

Référence électronique

Nathalie Roelens, «La question du roman terraqué aujourd'hui », Carnets [En ligne], Deuxième série -

19 | 2020, mis en ligne le 31 mai 2020, consulté le 23 décembre 2020. URL : http://

journals.openedition.org/carnets/11782; DOI : https://doi.org/10.4000/carnets.11782

Ce document a été généré automatiquement le 23 décembre 2020.

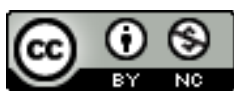

Carnets est mis à disposition selon les termes de la licence Creative Commons - Atribution - Pas d'utilisation commerciale 4.0 International. 


\title{
La question du roman terraqué aujourd'hui
}

\author{
Nathalie Roelens
}

Je songe à un proverbe portugais rapporté par

Gracq « Dieu écrit droit au moyen de lignes courbes. » (Ollivier, 1982 : 179)

\section{Introduction}

1 La géocritique se voit depuis peu confrontée à des injonctions « climatiques » venant de l'ecocritique qui jouit d'un nouvel élan depuis qu'elle a été relayée en France dans une visée éthique par Nathalie Blanc et Stéphanie Posthumus. Notre objectif n'est pas d'évaluer le mérite de ces courants mais d'ériger le roman «terraqué » - en l'occurrence breton - en laboratoire, afin d'éviter le double écueil que l'écocritique même dans ses plus beaux atours - discipline qui se donne pour tâche «d'analyser comment les représentations du monde peuvent refléter et influencer les façons dont l'humanité interagit avec les autres composants de l'écosphère (Sibley-Esposito, 2013 : 8) - suscite: d'une part, l'impératif référentiel qui mène à l'instrumentalisation (évaluer les textes selon des critères environnementaux purement thématiques) et, d'autre part, sa mythification et sa réoccupation politique (le penchant pour une littérature du terroir pastorale ou folklorique (retour un peu béat à la nature que l'on reproche à l'écologie). La solution nous semble résider dans une prise en compte de la complexité des mots, par le biais d'une sémiosis «transitive » (Barthes, 1957: 220), et des choses, par le biais d'une saisie d'un monde «fini et néanmoins foisonnant » (Caillois, $1970: 71$ ).

\section{Arbres, montagnes, coquillages}

2 Que l'humanité interagisse avec les autres composants de l'écosphère et que la littérature réinvente ce rapport, l'éthologue Jakob von Uexküll l'avait déjà pressenti en 
concevant le milieu (et non l'environnement) comme la cible de nos trajections, proche de ce que l'on appellerait aujourd'hui l'agentivité (affordance). Quel que soit l'environnement (Umgebung), un milieu (Umwelt) est nécessairement le plus adéquat pour l'espèce concernée, en harmonie avec ses « aptitudes ou facultés » (Uexküll, 2010 : 164). Une vache interprète l'herbe comme nourriture tandis que le chien lui trouve une autre fonction de sorte qu'ils ne vivent pas dans le même milieu même s'ils partagent objectivement le même environnement. Le "milieu» peut toutefois s'émanciper totalement des stimuli externes et devenir " magique ». Un chêne dans une forêt peut conférer une tonalité d'exploitation pour le forestier, une tonalité d'ascension pour l'écureuil, une tonalité de support pour les oiseaux chanteurs, mais une tonalité de danger pour la petite fille (l'écorce boursouflée qui ressemble fortuitement à un visage humain devient un démon.) (Cf. ibid. : 158)

Roland Barthes, dans «Le mythe aujourd'hui ", rejoint cette même saisie différenciée du monde en bon sémiologue qui distingue langage-objet et métalangage : «Si je suis un bûcheron et que j'en vienne à nommer l'arbre que j'abats, quelle que soit la forme de ma phrase, je parle l'arbre, je ne parle pas sur lui. Cela veut dire que mon langage est opératoire, lié à son objet, d'une façon transitive : entre l'arbre et moi, il n'y a rien d'autre que mon travail, c'est-à-dire un acte : c'est là un langage politique.» (Barthes, 1957 : 219). L'exemple de Barthes a beau sembler obsolète, prêter le flanc à tous les détracteurs de l'anthropocène, ce parler-arbre peut, semble-t-il, nous sortir de l'impasse où s'est engouffrée l'écocritique. Barthes y revient d'ailleurs vingt ans plus tard, dans le contexte du haïku et du satori zen qu'il affectionne. L'intraitable de la nature et le banal se recoupent en ce qu'il n'y a rien à en dire, qu'ils relèvent de l'exorbitant. Barthes cite le Wu-shi, une manière de déjouer la compulsion herméneutique occidentale, rappelant la parabole du maître zen Suzuki selon lequel il y a trois façons de regarder le monde: "les montagnes sont des montagnes; les montagnes ne sont plus des montagnes ; les montagnes redeviennent des montagnes. » (Barthes, 2003: 126) Le premier moment serait celui de la Bêtise, de la tautologie arrogante, un sou est un sou, etc.; le deuxième moment: celui de l'interprétation paranoïaque; le troisième moment, celui de la naturalité: «Ce processus : en quelque sorte, le retour de la lettre : le haïku (la phrase bien faite, la poésie) serait le terme d'un cheminement, l'assomption vers la lettre (...) la saisie de la naturalité de la chose » (ibid. : 127).

Dans Les Vacances de Monsieur Hulot de Jacques Tati (1953) - film terraqué si l'on veut -, une phrase du dialogue rare voire inexistant, «Oh un coquillage!», prononcée par la vacancière, tranche sur la thématique de l'invasion d'une petite station balnéaire (Saint-Marc-sur-Mer proche de Saint-Nazaire) par une société qui s'apprête déverser sur la côte ses rancœurs et ses mesquineries. 


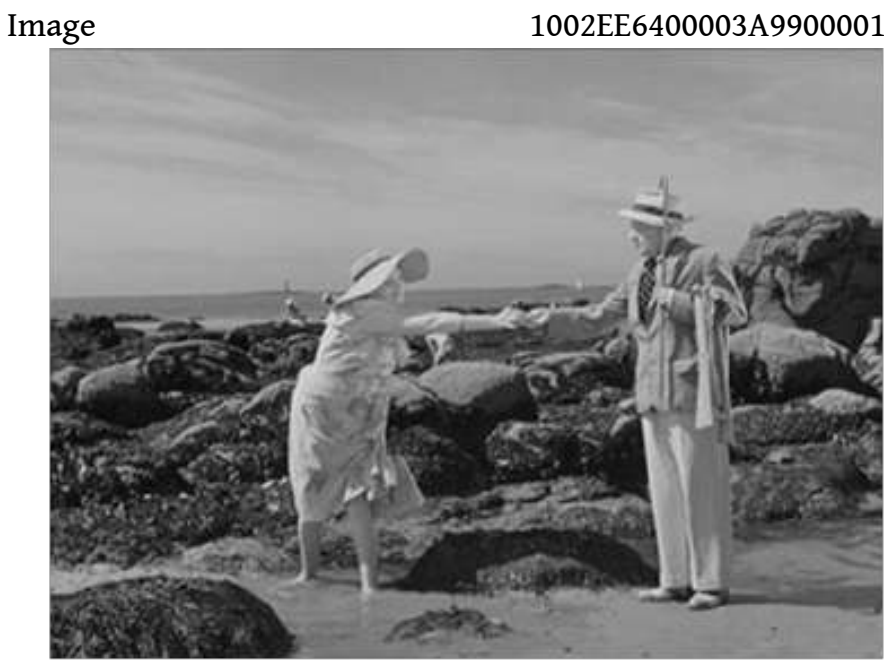

Oh! Un coquillage - Les vacances de M. Hulot

https://www.dailymotion.com/video/x1g3z12

5 Si l'on situe en effet l'humain dans l'écosphère, système d'interdépendances, composé de l'atmosphère, l'hydrosphère, la lithosphère et la biosphère, on restitue à l'individu son statut de corps vivant, d'entité naturelle, de zoé (« le simple fait de vivre, commun à tous les êtres vivants (animaux, hommes ou dieux)» (Agamben, $1997: 9)$ le dépouillant de son bios, forme de vie susceptible d'être assujettie à un pouvoir, à une biopolitique ou «Biocratie» (Debray, 2019 : 42). Et cela nous invite, dans le sillage de Bruno Latour qui appelle de ses vœux un « atterrissage » (Latour, $2017: 16$ ) après les dégâts causés par la mondialisation, à retrouver «le sens étymologique de la natura latine ou de la phusis grecque, que l'on pourrait traduire par processus, cours des choses » (Latour, $2017: 89$ ), « système d'engendrement » (ibid. : 112).

D'ailleurs, à en croire Clare Sibley-Esposito (2013), cette une réinsertion de l'homme dans la nature qui entraîne une remise en question du binôme Nature/Culture était déjà une des conquêtes épistémologiques de Roger Caillois, qui dans son "Nouveau plaidoyer pour les sciences diagonales» (Caillois, 1970:53-59) récuse la nette séparation des règnes, humain, animal, végétal et minéral, postulant un entrelacs d'interconnexions au sein de l'architecture du monde, ou « unité profonde ». Témoin, les similitudes inattendues entre les formes de certains objets fabriqués par l'homme et les manifestations déroutantes de ce qu'il appelle le «fantastique naturel ", telles que le visage fabuleux dans la livrée d'un papillon ou l'abdomen d'une araignée. Cette conception des « récurrences dérobées " repose sur l'hypothèse d'un monde fini (de nos jours le constat que les ressources sont limitées et que la perpétuation de la vie elle-même est tributaire d'une vision holistique du monde. Or, pour Caillois, si le monde est fini, il est également «foisonnant» (Caillois, 1970 : 71). Aussi celui-ci prônet-il une «méthode » poétique qui permettrait de «cerner l'insaisissable » et d'établir des « relations indirectes et justes » (ibid. : 254) entre les données du monde. En découle la conviction que certaines analogies fournies par l'imagination poétique puissent être «justes", révélant « une relation inattendue, une connivence nouvelle dans le réseau de l'inextricable univers» (ibid.: 218), comme la montagne de Barthes ou le coquillage de Tati. Évaluer la justesse d'images littéraires devrait être une des préoccupations de l'écocritique telle que nous l'entendons, «justesse » ne signifiant pas fidélité mais 
plutôt ancrage dans la complexité du monde, une façon de susciter le monde par le langage.

7 Ces longs préambules, entre Uexküll, Barthes et Caillois, étaient utiles pour réunir les conditions d'une écocritique revisitée à l'épreuve d'une immensité maritime et qui ne prêterait plus le flanc à des reproches de tropisme champêtre, rétrograde, fleur bleue.

\section{L'imaginaire terraqué}

Attesté depuis Voltaire, qui parle du globe terraqué dans son Memnon (1747), du latin terra et aquosus, composé de terre et d'eau, ou du bas latin, terraqueus, l'acception de «terraqué » comme épithète date des Travailleurs de la mer de Victor Hugo (1866), histoire de machination crapuleuse et d'amour, que celui-ci dédie pourtant au « rocher d'hospitalité et de liberté, à ce coin de vieille terre normande où vit le noble petit peuple de la mer, à l'île de Guernesey, sévère et douce, mon asile actuel, mon tombeau probable» (Hugo, $1975: 619$ ). Dans l'édition de 1883, Hugo adjoint une longue « ode à la mer », intitulée «L'Archipel de la Manche» dont le premier chapitre «Les anciens cataclysmes » donne le ton:

L'Atlantique ronge nos côtes. La pression du courant du pôle déforme notre falaise ouest. La muraille que nous avons sur la mer est minée de Saint-Valery-sur-Somme à Ingouville, de vastes blocs s'écroulent, l'eau roule des nuages de galets, nos ports s'ensablent ou s'empierrent, l'embouchure de nos fleuves se barre. Chaque jour un pan de la terre normande se détache et disparaît sous le flot. Ce prodigieux travail, aujourd'hui ralenti, a été terrible. Il a fallu pour le contenir cet éperon immense, le Finistère. Qu'on juge de la force du flux polaire et de la violence de cet affouillement par le creux qu'il a fait entre Cherbourg et Brest. Cette formation du golfe de la Manche aux dépens du sol français est antérieure aux temps historiques. La dernière voie de fait décisive de l'océan sur notre côte a pourtant date certaine. En 709, soixante ans avant l'avènement de Charlemagne, un coup de mer a détaché Jersey de la France. (ibid. : 563)

Pages visionnaires s'il en est, comme si Hugo était déjà en train d'expliquer la théorie des fractales de Mandelbrot qui prit précisément l'escarpement de la côte bretonne pour exemple et que les écologues ont invoquée pour expliquer l'effritement de celle-ci. Les autres chapitres évoquent les risques de la mer, légendaires ou réels, des vagues scélérates causant des naufrages ou, pire, les dangers de la mer tranquille, rôdeurs, orties infernales, paréidolies (mirages ou illusions d'optique) dont le granit est pourvoyeur, tout un bestiaire hideux prodigué par les rochers et les falaises. Le vocabulaire météorologique n'est pas en reste: «le singe, l'anuble, et le derruble. Le singe (swinge), c'est le courant ; l'anuble (lieu obscur), c'est le bas-fond ; le derruble (qu'on prononce le terrible), c'est le tourbillon, le nombril, l'entonnoir de roches sousjacentes, le puits sous la mer. » (ibid. : 570).

10 La suite du texte exhume des reliques de pratiques révolues à travers le présent de l'habitat et des usages :

D'autres cabanes ont été des barques ; une coque de bateau renversée, et juchée sur des pieux et des traverses, cela fait un toit. Une nef, la cale en haut, c'est une église ; la voûte en bas, c'est un navire ; le récipient de la prière, retourné, dompte la mer. Les bestiaux boivent dans des auges pareilles à des sarcophages. Un roi celte a peut-être pourri dans ce coffre de granit où s'abreuve paisiblement la vache, qui a les yeux de Junon. (ibid. : 573) 
11 Plus loin, c'est la « light-house » électrique qui a remplacé d'antiques brasiers à frissons de flammes suscitant toute une série de gestes désormais éteints.

Et, pour finir, Hugo insiste sur la bonté et la grandeur d'âme de ce noble petit peuple, cette race à part à qui il accorde une suprématie, par sa faculté de combiner l'oisiveté et le travail :

Ces peuples ont gardé de leur vieille vie de contrebandiers un goût hautain pour le risque et le danger. Ils vont partout. Ils essaiment. L'archipel normand colonise aujourd'hui, comme jadis l'archipel grec. C'est là une gloire. Il y a des jersiais et des guernesiais en Australie, en Californie, à Ceylan. (...) Où prospérait le banditisme, le commerce règne. (...) Là où ce peuple a été pirate, il est pilote. Ce pays est resté le pays de l'aventure en devenant le pays de la probité. (ibid.: 616-617)

Ce qui explique leur rapide promotion est le mouvement centrifuge de naviguer tous azimuts et, centripète, de revenir gros de l'expérience des antipodes, tel le flux et le reflux, vers " une ville d'arrivée et de départ, de passage et de retour » (Laurel, 2016 : 45), telle que Hermínia Laurel qualifie Lisbonne, ce Finisterre du sud, balcon sur l'infini, lieu d'escale, tremplin vers l'ailleurs, frange de l'Europe, particulièrement propice à croiser les focales (depuis la terre, depuis la mer). Nous avons eu l'occasion nous-même d'interroger les villes côtières (cf. Marrama, Roelens, 2016), animées d'une "morale naturelle» (Bachelard, 1993: 22), en prise avec une autre condition, dotées de porosité sociale et culturelle, ce qui favorise la mixité, façonne des êtres plus généreux et ouverts, mais offre aussi une certaine perméabilité à la mala vita qui s'infiltre dans les moindres recoins et niches urbaines, tandis que les villes continentales, plus conservatrices, recroquevillées seraient atteintes de "solipsisme " (Cassano, 1996). Cette dialectique recoupe en quelque sorte la théorie de la sémiosphère de Youri Lotman (1999) dans la mesure où le centre serait plus homogène, impliquant une adhésion majeure aux valeurs culturelles officielles, et la périphérie plus hétérogène, dissidente, novatrice.

13 L'ode à la mer hugolienne met le doigt sur trois points majeurs: (a) L'ambivalence entre bonté et amoralité, sacré et païen ; (b) la nature comme engendrement de formes, de techniques et de pratiques, pain béni pour les adeptes de la «morphogenèse des lieux »; (c) le parler-arbre, que ce soit la faune et la flore ou la connaissance de l'architecture navale.

\section{Trois romans bretons}

Dans les trois récits terraqués (Balzac, Genet, Ollivier), nous assistons à l'exacerbation des passions comme s'il y avait une homologie entre la géographie escarpée (fractale), les sensations fortes et le côté abrupt et rocailleux des mots en /k/ ou en /R/: « Le Croisic » chez Balzac; le «brick» chez Éric Ollivier (« Le brick, en langue maritime, c'était le similaire du bordel terrien. Mais le mot avait un son de vaisseau à sabords » (Ollivier, 1982: 60) ; "se brester » chez Jean Genet: "Se 'brester'. De bretteur sans doute: se quereller» (Genet, 1953: 12), jusqu'au surnom du protagoniste ou Brest même, "ville de brouillard et de granit » (ibid. : 10).

Les trois axes - (a) moral, (b) morphogénétique et (c) sémiologique - repérés chez Hugo se distinguent dans nos œuvres terraquées.

L'ambivalence bonté/amoralité se traduit, dans la nouvelle balzacienne Un drame au bord de la mer (1834), par une nature d'abord romantique, presque chateaubrianesque, 
qui accueille Louis et Pauline en villégiature au Croisic: "trois immensités qui nous entourent, l'eau, l'air et les sables" (Balzac, $2013:$ 35) - pays beau pour les grandes âmes, «il ne peut être habité que par des poètes ou par des bernicles. (ibid. : 34) -, et dont la rencontre d'un pauvre pêcheur parfait le tableau :

la profondeur muette de cette vie inconnue, admirant la noblesse de ce dévouement qui s'ignorait lui-même; la force de cette faiblesse nous étonna ; cette insoucieuse générosité nous rapetissa. Je voyais ce pauvre être tout instinctif rivé sur ce rocher comme un galérien l'est à son boulet, y guettant depuis vingt ans des coquillages pour gagner sa vie, et soutenu dans sa patience par un seul sentiment (ibid : 31)

Après le récit de l'histoire âpre et tragique (filicide) du stylite Pierre Cambremer, dont le nom rime avec «mer », «terrifiant et sacré à la fois » (ibid. : 11), la nature n'est plus accueillante mais redevient austère zone de non-droit, « un endroit que le fisc a jugé tellement inabordable que le douanier n'y passe presque jamais. » (ibid.: 24), sombre et dysphorique, comme frappé d'une terrible malédiction : « nous marchions au milieu de la nature la plus âcrement sombre que j'aie jamais rencontrée. Nous foulions une nature qui semblait souffrante, maladive; des marais salants, qu'on peut à bon droit appeler les écrouelles de la terre. » (ibid. : 53) Les marais salants sont les « écrouelles de la terre ", par où suintent les purulences du monde » (ibid. : 54)

À son tour, Querelle de Brest de Jean Genet (1953) exalte le crime, le vol et la pédérastie tandis que le brouillard lui accorde une impunité aux dockers et marins :

Les crimes sont à Brest aussi rares qu'ailleurs, mais par le fait du brouillard, de la pluie, du ciel épais et bas, de grisaille du granit, du souvenir des galériens, (...), par le fait de l'ancien bagne, du fil invisible mais solide qui relie les anciens marins, amiraux, matelots et pêcheurs aux régions tropicales, l'atmosphère y est-elle, lourde et cependant radieuse, qu'elle nous paraît nous seulement favorable mais essentielle à l'éclosion d'un meurtre. L'éclosion est le mot juste. Il nous semble évident qu'un couteau déchirant à n'importe quel endroit le brouillard, qu'une balle de révolver le trouant fissent, à hauteur d'homme, crever une outre et couler du sang le long des parois et à l'intérieur de ce mur vaporeux. Où qu'on frappe, le brouillard se blesse et s'étoile de sang. (Genet, $1953: 68$ )

Cet épais brouillard «criblé de poignards " (ibid. : 109), qui accueille le sang, le sperme (« un jet de foutre» (ibid.: 69)) et les larmes, adoucit en même temps les visages : «Tous les visages sont beaux, adoucis, purifiés par l'imprécision.» (ibid.), «brouillard déformant " à la Turner (Ollivier, 1982: 93) chez Ollivier. Qui plus est, la poésie, ellemême imprégnée de fluidité marine, d'une émotion qui déferle comme les vagues, allie la grâce au péché jusqu'à transfigurer ou innocenter les soi-disant délits et le stupre. Ainsi, la patronne de la Feria, le bordel de Brest, est-elle décrite comme « une perle océanienne parmi les nacres d'une huitre qui peut ouvrir sa valve quand elle veut, et la refermer aussi. » (Genet, 1953 : 29) Et « cet officier qui cherchait des bites » comme un pêcheur qui «dans les rochers, cherche l'anguille» (ibid.: 87). Querelle, dès lors qu'il revient de loin, « un de ses pieds repose encore sur une plage océanienne » (ibid.: 10) est enveloppé de nuées, nimbé d'une sorte de désintéressement, « comparable à l'ange de l'Apocalypse dont les pieds reposent sur la mer » (ibid.: 14). Le récit sublime même le fait qu'il ait dans plusieurs points du monde des dépôts secrets pour ses bijoux volés, car il se fait d'emblée allégorique: "Qu'ils descendent du ciel, ou remontent d'un domaine où ils connurent les sirènes et des monstres plus étonnants, à terre les marins habitent des demeures de pierres, des arsenaux, des palais dont la solidité s'oppose à la nervosité, à l'irritabilité féminine des eaux » (ibid.: 10), jusqu'à cerner le marin par oxymore « un joyeux suicide moral » (ibid. : 56 ). 
18 Et, enfin, dans L'orphelin de mer, où Éric Ollivier s'adonne à ce que Hermínia Laurel appelle une "géobiographie» (Laurel, $2017:$ 45), le narrateur retient de son enfance brestoise avant la mort du père, espèce de phare humain sur ce paysage terraqué qui entoure le jeune enfant, indissociable de la fascination pour la mer dont celui-ci est tantôt l'émissaire tantôt le nautonier, les moments de contact direct avec la puissance des éléments dans la demeure familiale vétuste, bâtie deux siècles plus tôt, presque au ras des flots: "Le nez littéralement collé pendant des heures contre la vitre sur laquelle venaient cingler les averses ou étinceler les coups de soleil, je ne me rassasiais jamais.» (Ollivier, 1982: 42) Les verbes "cingler» et «étinceler » sont comme des matrices de tout un vécu où les sensations sont exacerbées, un biotope que le texte relaie par l'usage d'hyperboles ou par des métaphores vigoureuses et chargées d'affect : «mes besoins de bourrasques» (ibid.: 120) «mon tropisme océanique» (ibid.: 178). Cette puissance contraste avec la "texture d'écume » (ibid.: 21) du père dont la mer était l'épouse poétique, "épouse primordiale ( (ibid.: 27), plus à l'aise avec les cormorans qu'avec les terriens, en empathie avec «tous les marins de la terre, au sens presque ésotérique du terme » (ibid. : 26), s'adonnant même à des offices, dont la scène primitive païenne à laquelle le jeune garçon assiste : son père étendu les bras en croix vers le ciel sur un tertre de granit, en symbiose panthéiste avec les éléments : «C'était un rite que célébrait fréquemment Arthur, c'était sa messe au soleil» (ibid.: 71) Cet aura magique qui nimbe le père se prolongera lors de son engloutissement final lorsque l'enfant a huit ans qui inaugurera malgré lui « l'excommunication de la mer » (ibid. : 11) de celui-ci. D'où l'étrange relation du fils avec l'océan, une attirance mêlée d'effroi : «j'ai ma théorie du naufrage : c'est lui qui est le prévisible, toute traversée paisible est l'exception » (ibid. : 30). Le clivage père aventureux, pirate dans l'âme/mère austère et pudibonde se traduit alors en un clivage entre deux peuples bretons, celui qui reste enclavé dans les terres de l'intérieur et n'avait pas bougé depuis des siècles, fermé sur ses coutumes et ses obscurités; celui des côtes, "ouvert à toutes les mers, hanteur de tous les continents, attiré par le large et les songes de déraison ». (ibid. : 26)

Tel le jeune Marcel chez Proust qui échappe à l'engluement familial par des escapades clandestines hors de Combray, ou le jeune Agostino chez Moravia qui s'initie auprès de voyous qui se retrouvent à l'extrémité de la plage toscane, le narrateur est attiré par le fruit défendu d'une meute de garçons, «chenapans sans bride » (ibid. : 57) ayant établi leur fief dans le quartier du port, sur les hauts tas de bois de construction débarqués du grand Nord, après avoir converti les poutres en balançoires. Ce fief éphémère indique la conversion d'usage d'un lieu qui là encore témoigne d'une vie hardie, tributaire des aléas et sans cesse reconfigurée par les marées: "J'aimais aussi explorer interminablement les criques et les grèves, découvrant els coquillages déposés par le flot, les épaves de navires traînées sur le sable par les fortes marées, et je rêvais vers les lointains. » (Ibid.: 63) Le jeune garçon assiste aussi à l'évolution urbanistique de vieux centre aux ruelles étroites qui, s'il n'avait été bombardé, serait devenu un quartier de rénovation «où se rueraient les snobs, les poutres apparentes et les décorateurs antiquaires. " (ibid.: 59) De même, les hôtels datant de la conquête des Indes sont délabrés depuis les conquêtes de la marine à vapeur et désormais convertis en bordels et, enfin, les cargaisons de tonneaux de rogue de Norvège, appâts pour la pêche à la sardine, qui enivrent par leur pestilence ses promenades au bord des quais : "une odeur d'algues, de pourriture discrète, de saumure, de goudrons et de bois aussi.» (ibid. : 57) 
Des lieux aussi mouvants sont en effet susceptibles d'une approche morphogénétique, qui étudie le processus dynamique entre strates, déterminant des nappes de sens: la « strate physico-symbolique » engendrant la strate « socio-culturelle intermédiaire » et à son tour celle de la "matérialisation effective des prégnances latentes, devenues des saillances » (Marcos \& Morier, 2019 : 556). Les productions culturelles feraient émerger la strate symbolique profonde par-delà les autres.

Chez Balzac, l'ampleur cosmique « ici, des sables; en haut, l'espace ». (Balzac, $2013: 34$ ), symbiose du végétal, animal, minéral, en éternelle métamorphose, enfante des rochers qui lèvent leurs têtes tels « des animaux gigantesques couchés dans les dunes » ou des récifs prenant « l'apparence de grandes roses blanches flottant sur l'étendue liquide et venant se poser sur le rivage ». (ibid. : 34) Les landes desséchées par le vent et les marais salants dénués de végétation, déterminent des pratiques commerciales bien précises : "les débris des embarcations hors de service se vendent aux riches, car le prix des transports les empêche sans doute de consommer le bois de chauffage dont abonde la Bretagne. N'a-t-il pas fallu que l'entrepôt du sel se plaçât sur ce rocher pour qu'il fût habité. » (Ibid.)

Dans Querelle de Brest, le bagne a cédé sa place à la marine marchande avec ses entrepôts et ses docks, à tel point que les mousses s'avèrent « la progéniture monstrueuse, délicat et débile des bagnards accolés et accouplés (Genet, 1953 : 101). De même, le double écusson de France et de Bretagne qui orne la façade du bagne désaffecté a perdu son « autorité fécondante » (Ibid. : 100) : «La convexité du double écusson ne signifie plus rien. Elle ne correspond plus au gonflement des voiles, à la courbe des coques de bois, à la gorge orgueilleuse des figures de proue, aux soupirs des galériens, à la magnificence des combats navals. » (Ibid. : 101)

L'orphelin de mer réactive à son tour les couches enfouies. Il passe du temps sur des bateaux abandonnés dans d'anciens chantiers navals désaffectés, avec ses carcasses de navires en fer. Et il explique que jusqu'à la fin du XIX ${ }^{e}$ siècle la Bretagne entretenait des forêts dont les meilleurs arbres étaient réservés pour la construction des navires. Le bois était la prospérité. Comme si le bois avec sa sève était plus en phase avec l'esprit des habitants de la mer au contraire du fer, matériau venu d'ailleurs : « Les bateaux en bois disparaissaient trop vite, se désintégraient en peu d'années, ne laissant plus que d'énormes squelettes, d'immenses armatures de poissons morts où habitaient crabes et crevettes. Or, la pèche n'intéressant pas les navigateurs que nous étions par vocation, c'est aux flottes de métal que nous donnions l'abordage. » (Ibid. : 61)

Le parler-arbre doit être entendu ici au sens de climat onomastique, atmosphérique. Il se traduit dans nos trois textes par des géolectes ou océanolectes, termes que nous aimerions forger sur idiolecte, qui se distinguent d'un jargon pittoresque, en ce que lecteur ne possède pas forcément l'encyclopédie nécessaire à l'ameublement du monde représenté, contrairement aux images d'Épinal souvent tautologiques. Philippe Hamon nous a appris que l'inventaire du monde requiert une description paradigmatique et une ventilation syntagmatique, un travail sur l'onomastique jusqu'à l'amplification 
lexicographique et la dispersion anagrammatique (par exemple du mot "mer») (Cf. Hamon, 1986)

Chez Balzac, sans aller jusqu'à emprunter à la langue bretonne, la couleur locale passe par l'usage de termes locaux populaires «l'iraigne » pour l'araignée (Balzac, $2013: 28$ ), «paludiers, nom donné à ceux qui cultivent le sel » (ibid. : 54) et des allusions aux vieux métiers : « la pèche aux engins ou à la ligne ", « porter du sel sur le port " «travailler aux marais salants", voire aux habitudes culinaires «Oh! monsieur, nous mangeons des galettes de sarrasin et des bernicles que je détache des rochers » (ibid. : 29-31)

Querelle, transfiguré par le crime, comprend les choses sans intermédiaire dans leur singularité - la montagne est redevenue une montagne - : «Accroupi dans le velours noir des herbes, des arums, des fougères, dans la nuit vivante de son intime océanie, il garde les yeux grands ouverts. » (Genet, 1953 : 141). Il a atteint un rapport élémentaire avec la materia prima du monde, ou mer-terre.

Le père taciturne de "l'orphelin » connaît pourtant, à la grande surprise de son fils «toutes les chansons de marine où Valparaiso et quelques autres mots bariolés revenaient fréquemment tempérer les mélancolies chroniques de ces mélodies " (Ollivier 1982 : 23). Incorporés aux phrases du quotidien, se mêlent « les noms les plus exotiques. Ils chantaient comme au fond d'un coquillage collé à l'oreille ; Mourmansk, Yokohama, Salonique, Londres, Gibraltar. » (ibid. : 27) Mais aussi « un répertoire d'airs bretons que je croyais ensevelis à jamais, (...) un mélange de cantiques approximativement chrétiens (La Bretagne était encore solidement païenne au XIX ${ }^{\mathrm{e}}$ siècle, malgré les apparences ), de complaintes d'amours malheureuses » (ibid. : 24) On a droit aussi à des vestiges de métiers disparus qui ponctuent le rythme de la cité : les vidangeurs, "rats à cloaques", des trônes, "larges fauteuils plats, en bois sombre, à cuvette de porcelaine » (ibid. : 81), les ramasseurs de «bourrier »: « Le bourrier, sur la côte atlantique, c'est le nom rustique des ordures ménagères » (ibid.) qui sert à fumer les terres pauvres, les marchandes de laits (déjà chez Proust), etc.

\section{Pour une écocritique re-localisée}

La bifrontalité inhérente aux bords de mer, la réalité portuaire oxymorique, bref le style de vie terraqué serait l'antidote contre la vieille idéologie du sang et du sol (Blut und Boden) d'une littérature "localiste " et aux connotations conservatrices que le retour « vers la glèbe " (Debray, 2019:50) suppose. Consterné par la « dénégation de la mutation climatique » (Latour, $2017: 30$ ), Latour invite à « déglobaliser » (ibid.: 128), à « rediriger l'attention de la 'nature' vers le Terrestre » (ibid. : 105), d'une part, contre la fuite en avant imposée par la modernisation et, d'autre part, contre l'attachement au sol comme maintien d'une tradition ancestrale, native, d'une "nostalgie pour des positions 'archaïques' ou 'obscurantistes » (ibid. : 25). Il invite à relocaliser la question du global en multipliant les points de vue, les variétés, à rebours d'une seule vision tout à fait provinciale qui s'est imposée à tous et répandue partout : «Alors que le local est fait pour se différencier en se fermant, le Terrestre est fait pour se différencier en s'ouvrant» (ibid.: 72). Pour Latour c'est le déracinement qui est illégitime, non l'appartenance. Il conviendrait selon lui de réinventer un terroir «après que la modernisation a fait disparaitre tous les anciens attachements. C'est un Local par contraste. Un anti-Global » (ibid. : 39). 
Nous souscrivons entièrement à cette proposition d'autant plus que les villes portuaires sont aux premières loges en matière de dégâts subis par la mondialisation. Le récitdocument de Saviano Gomorra nous montre une ville portuaire comme endroit autrefois chaleureux et bruyant, peuplé d'hommes cousus de cicatrices et parlant des langues improbables, qui se voit désormais muselée par une économie mondialisée, au service d'un mafia internationale insaisissable, en proie à la fraude légalisée de la grande finance. Le port est devenu une gigantesque excroissance de la ville, lieu de transit totalement désolidarisé du vécu urbain, usine automatisée qui métabolise des marchandises invisibles, branché sur des réseaux de transport et des routes commerciales illégales (renversement pathétique de la route des épices ou de la soie). (cf. Saviano, 2007).

Le roman terraqué comme condensé du destin de notre globe terraqué, avec ses pentes vicieuses et ses gestes vertueux, peut enjoindre notre culture à atterrir et à amerrir/ accoster. L'écocritique devrait tourner sa lorgnette vers le petit, le menu, l'être-là des choses les plus frêles, devenir miniaturiste du lieu, l'appréhender comme un énorme réservoir en pratiques et en poésie, non pas au sens d'une attitude verte de façade, ce que Régis Debray appelle avec malice le « retour à la chlorophylle, aux papillons et au bocage » (Debray, 2019: 43), ou encore "l'écolatrie» (ibid.: 51) des coquelicots : «Les cadres quittent le métro-boulot-caveau pour s'en aller baratter, biner, récolter, faucher, désherber - la vie au grand air incarnant la vie bonne.»(ibid.), mais respectueux des gestes que le lieu lui-même nous dicte: "Siliceuse ou argileuse, la nature d'un sol dicte un genre de semis, un mode de vie et de penser, avec un certain calendrier à respecter, qui ne dépend pas de nous. On doit semer le potiron d'avril à mai, dans un sol frais et ameubli (...)» (ibid. : 55). La montagne redevient une montagne et le sujet retrouve le chemin familier de ses trajections. L'acquiescement au monde est une épreuve d'humilité permettant les retrouvailles avec un monde fini mais néanmoins foisonnant, propice à l'éclosion de produits culturels inédits : «ce ne sont pas les idées que j'aime, mais les données du monde, les appels des larges pans de ténèbres derrière lesquels l'univers dérobe vite ses lois et ses manières, la pénombre où il dissimule les modèles de ses rouages comme la source des énergies qui les meuvent. » (Caillois, $1970: 8$ ).

\section{BIBLIOGRAPHIE}

AgAMBEN, Giorgio (1997). Homo sacer, vol. I, Le Pouvoir souverain et la vie nue. Paris : Seuil.

Bachelard, Gaston (1993). L'eau et les rêves (1942). Paris : Livre de poche « bibilo/essais ».

BALZAC, Honoré de (2013). Un drame au bord de la mer (1834). Rennes : La part commune.

BARTHES, Roland (1957). « Le mythe aujourd'hui » (1956) in Mythologies. Paris : Seuil.

BARTHES, Roland (2003). La préparation du roman (1978-1980). Paris : Seuil. 
BLANC, Nathalie, CHARTIER, Denis \& PUGHE, Thomas (2008). « Littérature et écologie : vers une écopoétique ", Écologie \& Politique. Éditions Syllepse, n³6, p. 17-28.

CAILloIs, Roger (1970). Cases d'un échiquier. Paris : Gallimard.

CASSANO, Franco (2005). Il pensiero meridiano. Bari : Laterza.

DEBRAY, Régis (2020). Le siècle vert. Un changement de civilisation. Paris : Gallimard « Tracts ».

GENET, Jean (1953). Querelle de Brest. Paris : Gallimard, «L'imaginaire ».

HAMON, Philippe (1986), Introduction à l'analyse du descriptif. Paris : Hachette.

HUGO, Victor (1975). Les travailleurs de la mer. Paris : Gallimard « Pléiade ».

JULLIEN, François (2014). Vivre le paysage ou L'impensé de la Raison. Paris : Gallimard.

LATOUR, Bruno (2017). Où atterrir ? Comment s'orienter en politique. Paris : La Découverte.

LAUREL, Maria Hermínia (2016). « Lire la ville au fil du temps. Lisbonne revisitée », in Nathalie Roelens \& Thomas Vercruysse. Lire, écrire, pratiquer la ville. Paris : Kimé, pp. 43-65.

LOTMAN, Youri (1999). La Sémiosphère, trad. Fr. Anka Ledenko. Limoges : PULIM.

MARCOS, Isabel \& MORIER Clément (2019). « Pour une sémiotique morphodynamique.

Sémiophysique de la frontière, in Amir Biglari \& Nathalie Roelens, La sémiotique et son autre.

Paris : Kimé.

OLLIVIER, Éric (1982). L'orphelin de mer...ou les mémoires de monsieur de Non. Paris : Denoël.

POSTHuMus, Stéphanie (2011). « Vers une écocritique française : le contrat naturel de Michel

Serres ». Mosaic : An Interdisciplinary Critical Journal, vol. 44, No. 2, pp. 85-100.

ROELENS, Nathalie, MARRAMA, Christabel (2017). «Éthique de la ville. Entre villes-côtières et villes-

forteresses : un paradigme paradoxal ", in Carole Bisenius-Penin, Lieux, littérature et médiation dans

l'espace francophone. Metz : PUN, Éditions Universitaires de Lorraine, pp. 125-138.

SAVIANO, Roberto (2006). Gomorra. Paris : Gallimard, 2007.

SIBLEY-ESPOSITO, Clare (2013). « Caillois sur les chemins de l'écocritique », Littératures, 68 [en ligne] [disponible le 29/4/2020] <URL : https://journals.openedition.org/litteratures/108>.

UEXKÜLL, Jakob von (2010). Milieu animal et milieu humain (1934). Paris : Payot \& Rivages.

\section{RÉSUMÉS}

Nous nous proposons de montrer en quoi le roman terraqué peut ouvrir des pistes à l'écocritique soupçonnée d'être l'auxiliaire d'une littérature du terroir, du pittoresque ou du primitif. L'entrelacs terre-mer engendre des œuvres qui, en confrontant deux imaginaire, deux morales, des coutumes terrestres ancestrales et l'imprévisible marin, lancent des défis d'ordre sémantique et éthique à l'écocritique, lui donnent une nouvelle légitimité à l'abri de toute récupération localiste. Trois romans bretons serviront de laboratoire à une écologie de l'esprit, dont les implications épistémologiques débordent le littéraire, nous engageant à voir la réinscription poétique dans le lieu comme une manière plus responsable, «opératoire " (Barthes) d'appréhender le monde qui nous environne, monde « fini et néanmoins foisonnant » (Caillois), en voie de « déglobalisation » (Latour). 
The aim of this contribution is to show how the terraqueous novel can open paths to an ecocriticism often tainted with a reputation of auxiliary to localist, picturesque or "wilderness" literature. The interlacing of land and sea generates texts which, by confronting two imaginaries, two morals, ancestral land customs and the unpredictable sea, launch semantic and ethical challenges to ecocriticism, gives it a new legitimacy far from any localist recovery. Three Breton novels will serve as a laboratory for an ecology of the mind, whose epistemological implications go beyond the literary, committing us to see the poetic re-inscription in the place as a more responsible, "operational" (Barthes) way of apprehending the world which surrounds us, a world "finished and nevertheless abundant" (Caillois), in the process of "deglobalization" (Latour).

INDEX

Keywords : terraqueous novel, ecocriticism, Caillois (Roger), Barthes (Roland), Latour (Bruno)

Mots-clés : roman terraqué, écocritique, Caillois (Roger), Barthes (Roland), Latour (Bruno)

\section{AUTEUR}

\section{NATHALIE ROELENS}

Université du Luxembourg

Nathalie.roelens[at]uni.lu 